\title{
An Energy Efficient Cluster Formation and Maintenance Scheme for Wireless Sensor Networks
}

\author{
A. S. M. Sanwar Hosen ${ }^{1}$, Seung-Hae $\mathrm{Kim}^{2}$, and Gi-Hwan Cho ${ }^{3^{*}}$, Member, KIICE \\ ${ }^{1}$ Division of Electronics and Information Engineering, Chonbuk National University, Jeonju 561-756, Korea \\ ${ }^{2}$ Division of Supercomputing Center, KISTI, Deajeon 305-806, Korea \\ ${ }^{3}$ Division of Computer Science and Engineering, Chonbuk National University, Jeonju 561-756, Korea
}

\begin{abstract}
Nowadays, wireless sensor networks (WSNs) comprise a tremendously growing infrastructure for monitoring the physical or environmental conditions of objects. WSNs pose challenges to mitigating energy dissipation by constructing a reliable and energy saving network. In this paper, we propose a novel network construction and routing method by defining three different duties for sensor nodes, that is, node gateways, cluster heads, and cluster members, and then by applying a hierarchical structure from the sink to the normal sensing nodes. This method provides an efficient rationale to support the maximum coverage, to recover missing data with node mobility, and to reduce overall energy dissipation. All this should lengthen the lifetime of the network significantly.
\end{abstract}

Index Terms: Wireless sensor networks, Routing, Clustering, Energy efficiency

\section{INTRODUCTION}

The vast potential applications of wireless sensor networks (WSNs) have attracted extensive research efforts. Conventional networks support mostly point-to-point or point-to-multipoint data forwarding [1].

On the other hand, WSNs are often deployed to sense, process, and disseminate information on targeted physical environments. Furthermore, sensor nodes are deployed in large numbers and high density, thus resulting in a potentially huge amount of sensory data, which is often impractical to transmit to the sink directly, particularly from the perspective of energy conservation. Also, the deployed sensors are capable of communicating with each other across short distances. Therefore, data aggregation is required with cluster formation to effectively gather the data from the widespread sensors, and a hierarchical structure is needed to forward the data to the sink in order to curtail the network load and hence reduce energy consumption $[2,3]$.

The traditional topology-based WSN routing protocols are quite susceptible to node mobility mainly due to the predetermination of an end-to-end route before data transmits.

Owing to the frequent and constant changing of the network topology, it is actually difficult to maintain a deterministic route. Therefore, link failure discovery and recovery procedures also consume time and energy.

The most critical requirements of WSNs are achieving the expected throughput as well as covering the entire network efficiently. According to some previous studies on WSNs, it is a challenging job to maintain the mobile sensors in network construction and data forwarding. The flooding/ multicasting technique is an efficient approach to broadcasting the control/data packets in an environment

Received 10 April 2012, Revised 05 June 2012, Accepted 12 June 2012

*Corresponding Author E-mail: ghcho@chonbuk.ac.kr 
where neither the sensor nodes nor the sink are stationary. However, it poses serious new challenges (i.e., idle-listening in the media access control (MAC) layer and control packet overhead) for WSN applications in which the constituted nodes move around.

For the above reasons, sensor nodes are prone to huge computational costs, which result in frequent death of some deployed nodes. The frequent death of nodes can bring about a link failure and/or partition in the network. In such cases, network reconstruction and maintenance must occur within a short period of time. This tends to bring about high energy consumption, which also degrades the network lifetime. To resolve this well-known problem, the most common approach is to make use of a hierarchical and clustering approach in the network construction and data forwarding.

The scheme presented in this paper differs from the conventional clustering approaches, and is designed to satisfy the requirements of node mobility, as follows.

1) Our scheme constructs the network based on a hierarchical approach (e.g., from source to destination) to distribute the tasks to avoid a huge computational cost to a specific node.

2) The control node election and the data routing both are follow a hierarchical approach that will reduce the control/data packet overhead in the whole network.

3) The periodic change of specially functioning nodes will support overcoming link failure/network partition due to the death of intermediate nodes in communication links.

4) Also, our scheme includes a method for collecting the missing data from a missing node that comes from out of the communication range.

This work presents an energy-efficient cluster formation and maintenance (ECM) scheme for WSNs based on a hierarchical approach. The initial construction of the network will be initiated from the sink by electing gateway nodes (GNs) in the entire network. The elected GNs will participate in cluster head $(\mathrm{CH})$ election to distribute the network area into several logical clusters. The elected $\mathrm{CHs}$ among the member sensor nodes will form an individual logical cluster. All these procedures are maintained as a top-down process from the sink to the normal sensor nodes. The normal sensor nodes, which are members of their corresponding $\mathrm{CHs}$, will sense data from their own environment and forward it to the $\mathrm{CHs}$. The $\mathrm{CHs}$ take a role of aggregator of the sensed data, and are responsible to forward it to the GNs. Finally, the GNs will transmit it to the sink based on the current location of sink. Therefore, the data forwarding will be maintained in the bottom-up procedure.
The remainder of this paper is organized as follows. We briefly discuss the works related to the routing vs. energy consumption point of view in Section II. In Section III, our proposed scheme is described in detail. Section IV shows the simulation results. Finally, Section V concludes our work.

\section{RELATED WORKS}

There are various routing protocols used for WSNs, but they are commonly application-specific [4]. Some of them are proactive and designed for static networks like DSDV [5], some are reactive and designed for mobile networks like DSR [6], and some are hybrid protocols like AODV [7]. Most of them are not suitable to directly apply to WSNs due to the high computational cost. In order to resolve this problem, there are several works based on hierarchy and clusters as follows.

LEACH [8] is a hierarchy and clustering-based protocol for periodic data gathering applications in WSNs. Sensor nodes elect themselves as $\mathrm{CHs}$ at any given time with a predefined probability. While these $\mathrm{CHs}$ advertise their status, the member nodes determine which cluster they want to join based on strength of each received signal. Nevertheless, the probability that there is only one $\mathrm{CH}$ or there is no $\mathrm{CH}$ is high when the desired number of $\mathrm{CHs}$ is small. Thus, when no $\mathrm{CH}$ is elected, all nodes must send data to the base station directly, which is not suited for short distance-capable tiny sensor nodes.

PEGASSIS [9] is a chain-based protocol that allows only one node (the leader) to transmit data to the sink in each round and requires the other nodes to instead transmit only to nearby neighbors. The key idea of this scheme is chaining and fusion. To construct a chain, each node determines its closest neighbor and forms a chain by employing a greedy algorithm. However, this protocol is not preferable for mobile nodes. Moreover, link failure/network partition can occur due to the death of an intermediate node in the chain.

DLER [10] is based on the idea that the sink elects a set of landmarks in the network to route data from the sensing field to the sink. To elect the landmarks, three different transmission ranges are used to broadcast control packets. After completion of the landmarks' election, the normal sensor nodes forward their sensed data using the greedy forwarding method based on the neighboring landmark information. However, this protocol is based on a static scenario of the constituted nodes, which does not take into consideration the energy level of a node determining whether it is a landmark. The different transmission ranges in each landmark election process dissipate more energy, which may lead to the quick death of those landmarks not long afterward. Moreover, the least number of landmarks near the sink may cause the dropping of the significant number of data packets from distant landmarks. 
EER [11] is a hierarchy and clustering-based protocol that can support mobile sensor nodes as well as mobile sinks. To solve the mobility issue of sensor nodes, this protocol makes use of two $\mathrm{CH}$ nodes and a GN is required in each cluster, where $\mathrm{CHs}$ play the role of an aggregator for data from their sensor nodes and transmit data through the GN. Eventually, the GN will transmit the aggregated data to the sink. However, this protocol violates the conventional definition of a cluster, where one $\mathrm{CH}$ should be elected in a cluster. Thus, it is not an efficient approach to elect more than one $\mathrm{CH}$ in a cluster, and arbitrate their corresponding roles. This means that it required more control packets, which induces a greater computational cost and consequentially degrades the network lifetime significantly.

The witness of the previous works shows that they emphasized constructing a network based on routing efficiency rather than overall energy dissipation, where the practical WSN application deserves to take on the last sensor node's functionality during the expected network lifetime.

\section{PROPOSED ENERGY EFFICIENT CLUSTER AND MAINTENANCE SCHEME}

Our work aims to construct a network from an energyefficient perspective, and to provide data delivery safely from the deployed sensor nodes to the sink. To begin with, it is based on the following assumptions:

1) The ID of a node is unique.

2) Nodes are homogeneous and energy constrained.

3) Nodes are mobile and able to adjust their transmission range.

In addition, we make use of the basic time division multiple access (TDMA) method in the MAC layer of the constituted nodes. After completion of the network construction phase, all the deployed sensors will maintain their allocated TDMA schedule. Data transmission from the normal sensor nodes to the $\mathrm{CH}$, from the $\mathrm{CH}(\mathrm{s})$ to $\mathrm{GN}$, and finally from the GN to the sink would be according to their own TDMA schedule.

The proposed scheme consists of three phases. The first phase is the initial network construction phase, where the sink initiates to elect GNs in the network. The elected GNs take charge of electing $\mathrm{CHs}$, where a $\mathrm{CH}$ corresponds to each logical sub-region of the network. In addition, the GNs transmit data delivered from their relevant $\mathrm{CH}(\mathrm{s})$ to the sink. The CHs will form logical clusters exclusively based on the information from the normal sensor nodes provided by the GNs. Also, the CHs take on the role of the aggregator of the data from their member sensor nodes and transmit the data to the sink through the GNs.
In the second phase, which is the data forwarding phase, the deployed sensors will start to accumulate information from their surrounding environments and transmit the data to the sink using their corresponding intermediate nodes (i.e., $\mathrm{CH}$ and $\mathrm{GN}$ in sequence). The last phase is called a network maintenance phase. Along with the mobility of the constituted node, the initial network structure will be broken, so network maintenance and reconstruction is required for an individual cluster or the entire network.

\section{A. Initial Network Construction Phase}

This phase performs the setup of an efficient hierarchical structure of the network. It defines the special purpose nodes, such as the GNs and CHs, and the logical clusters in order to build a network hierarchy. In the election process, the highest energy level and lowest mobility of nodes are preferable. Each step of the initial network construction phase is as follows.

\section{1) Gateway Nodes (GNs)}

The sink first initiates the election process by electing a set of GNs from among the normal sensor nodes. The sink broadcasts a $<$ hello $>$ message along with setting a timer $t_{s}$, where $t_{s}$ is the sending time. On receiving <hello $>$ messages from the deployed sensor nodes, they send back $<$ ack $>$ messages to the sink with <node_id, node_loch, node_energy, node_velocity $>$ within the time $t_{s}$, where node_id is the unique ID of a node, node_loch is the current location, node_velocity is the node's velocity, and node_energy is the remaining energy of a node. When the timer is expired, the sink will short out the received list to compute the highest energy level of nodes and the distance between GNs. If the distance is $R \leq \operatorname{dist}(i, j) \leq 2 R$, the sink will select $G N_{i}$ and $G N_{j}$ as GNs, where $R$ is the transmission range in between two adjacent nodes. Otherwise, the sink will select only the highest energy level of nodes $G N_{i}$ within $R$. After electing the GNs, the sink will inform the constituted sensor nodes of the information from elected GNs to the constituted sensor nodes and the allocated time slots for each GN to communicate with the sink.

\section{2) Cluster Head (CH) Nodes}

The elected GNs participate in processing the $\mathrm{CH}$ node election. Each GN broadcasts the $<$ hello $>$ message to the normal sensor nodes using the transmission range (3/4) of $\operatorname{dist}(i, j)$, along with setting a timer $t_{g}$. The nodes within the transmission range receive the message send back <ack $>$ messages with <node_id, node_loch, node_energy, node_velocity>. A GN will receive the messages from the nodes in the intersection and non-intersection region within the time $t_{g}$. The initiating $\mathrm{GN}$ tries to elect a $\mathrm{CH}$ 
node among the sensors in the non-intersection region. The GN with the highest energy level will elect the $\mathrm{CH}$ node in the intersection region first. The elected $\mathrm{CH}$ nodes will be the members of their initiator GN. When the timer is expired, the GN broadcasts the information of the elected $\mathrm{CH}$ nodes and their member sensor nodes, and assigns the allocated time slot for each member $\mathrm{CH}$ to communicate with the GN. The CH node then creates a TDMA based on the provided information from the GN and broadcasts it to the member sensor nodes. Finally, each normal node adjusts its transmission range to communicate with the $\mathrm{CH}$ node.

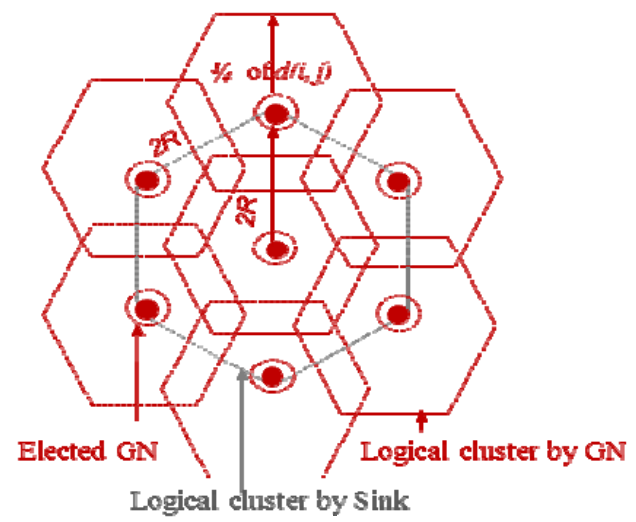

Fig. 1. An example of a well-formed cluster of GNs.

\section{3) Logical Cluster Formation}

The entire network would be distributed into logical clusters. The lower level clusters are grouped by normal sensor nodes and a $\mathrm{CH}$ node. The upper level clusters are grouped by $\mathrm{CH}$ node(s) and a GN, where logical clusters are connected hierarchically for bi-directional communication.

1) Logical cluster formed by the sink: This cluster is initiated by the sink to cover the entire network by elected GNs based on their energy level and low mobility. Fig. 1 shows an example of a well-formed cluster of GNs.

2) Logical cluster formed by the GN: The elected GNs formed the logical clusters by electing $\mathrm{CHs}$ in subregions. Fig. 2 shows an example of a well-formed cluster of CHs.

3) Logical cluster formed by $\mathrm{CH}$ : The elected $\mathrm{CH}$ forms a logical cluster with the normal sensor nodes. Fig. 3 shows an example of a cluster of normal sensor nodes and the overall network hierarchy.

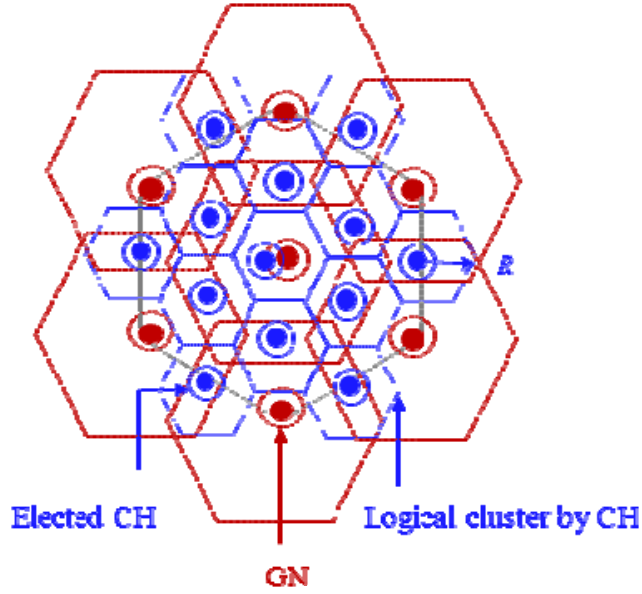

Fig. 2. An example of a well-formed cluster of $\mathrm{CHs}$.

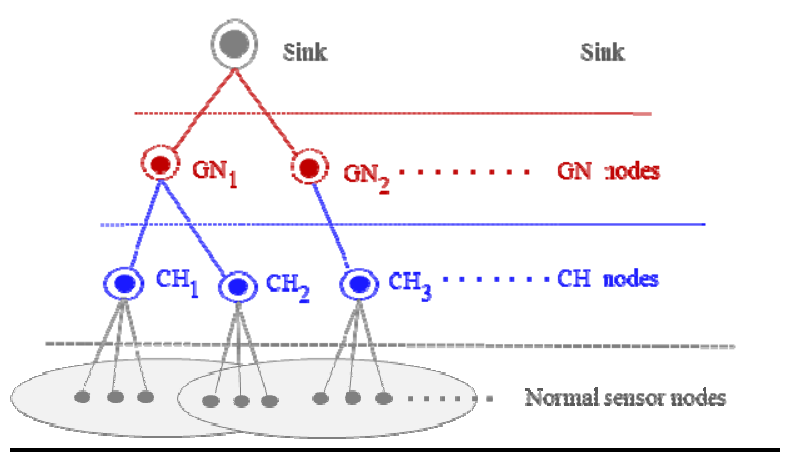

Fig. 3. An example of a cluster of normal sensor nodes and the overall network hierarchy.

\section{B. Data Forwarding Phase}

After establishing the network hierarchy, the normal sensor nodes will be initiated to begin sensing data from their surrounding environment and transmitting it to their corresponding $\mathrm{CH}$ in the assigned allocated time. The $\mathrm{CH}$ will accumulate the data from the different member nodes and transmit the aggregated data through the respective $\mathrm{GN}$ in their allocated time. The GN is also an accumulator of data from the member $\mathrm{CH}(\mathrm{s})$ and transmits data to the sink directly or in a multi-hop fashion. Thus, the sensed data will be gathering at the sink based on a bottom-up process.

\section{Network Maintenance Phase}

The logical structure of a cluster will be broken with constituted nodes' movements, when they go out of transmission range and/or node death of intermediate nodes occurs. This mobility feature worsens the efficiency of the 
proposed scheme. Therefore, two kinds of network maintenance, that is, local maintenance and network reconstruction are required.

1) Local maintenance: The network efficiency in our scheme is significantly degraded due to the mobility of sensors. If a $\mathrm{CH}$ node does not receive any data from a member sensor node within the pre-defined time, then the $\mathrm{CH}$ will inform the corresponding $\mathrm{GN}$ of the fact that the sensor node is missing. Of course, double-checking to verify node death is necessary. The GN node will broadcast a $<$ hello $>$ message to ask the node about its current status, especially its current location, and request to send the remaining data within a time $t_{m}$. On receiving the $<$ hello $>$ message, the corresponding node sends back an $<$ ack $>$ message with the remaining data and current location to the initiating GN within the assigned time. After receiving the $<a c k>$ message, the GN will compute the distance from the node's respective $\mathrm{CH}$ node. If the distance is within the communication range, the GN will inform the normal sensor nodes that the $\mathrm{CH}$ is alive, and tell the $\mathrm{CH}$ node to re-assign the time slot for the missing node, or else it will inform the missing node to stop the transmission to the $\mathrm{CH}$, Then, the $\mathrm{CH}$ eliminates the node from its member list. Otherwise, the node will be considered to be a dead node.

2) Network reconstruction: This is needed for the network in such situations where the average residual energy of $\mathrm{GNs} / \mathrm{CHs}$ is below a pre-defined threshold. Network reconstruction is nearly the same as the initial network construction. Basically, the network reconstruction will be maintained hierarchically, where the sink is the initiator to re-elect the GN node for a particular GN cluster or all of the GNs in the network, and each re-elected GN will be an initiator to re-elect $\mathrm{CH}$ node(s) within the $\mathrm{CH}$ member cluster. Similarly, each re-elected $\mathrm{CH}$ forms a cluster with the normal sensor nodes.

\section{PERFORMANCE EVALUATION}

We used a network simulator, ns2 [12], which is a discrete event simulator. The primary performance measurement is the increased network lifetime due to energy awareness during network construction and data routing. Moreover, we are interested in how this comparative measure scales with the network size. A WSN may involve thousands of nodes, thus scaling to a large network size is essential for a routing protocol to be applicable to sensor networks.

\section{A. Network Model}

To represent the sensors' mobility, we used the random waypoint model, which is similar to CBR [13]. Each mobile node moves at the direction of $0^{\circ}$ to $360^{\circ}$ to change the current location for a distance $d$ with a velocity between [velocity_min, velocity_max] where $d$ is exponentially distributed. The sensors will be reflected when they hit the boundary of the designated network area.

In order to calculate the transmitting and receiving energy, we used the first order radio dissipation model, which is also described in [13] as shown in Fig. 4. In this model, the transmitter and receiver dissipate $E_{\text {elec }}=50 \mathrm{~nJ} / \mathrm{bit}$ to run the transmitter and receiver. To amplify the signal, amplifier dissipates $E_{a m p}=100 \mathrm{pJ} / \mathrm{bit} / \mathrm{m}$ and the idle-listening dissipates $330 \mathrm{~mJ} / \mathrm{slot}$. Eqs. (1) and (2) in [13] are used to calculate the energy in transmitting and receiving a $k$-bit message through a distance $d$ between the transmitter and the receiver.

$$
\begin{aligned}
& E_{T x}(k, d)=E_{T x-\text { elec }}(k)+E_{T x-a m p}(k, d) \\
& E_{T x}(k, d)=E_{\text {elec }} \times k+E_{\text {amp }} \times k \times d^{2} \\
& E_{R x}(k)=E_{R x-\text { elec }}(k) \\
& E_{R x}(k)=E_{\text {elec }} \times k
\end{aligned}
$$

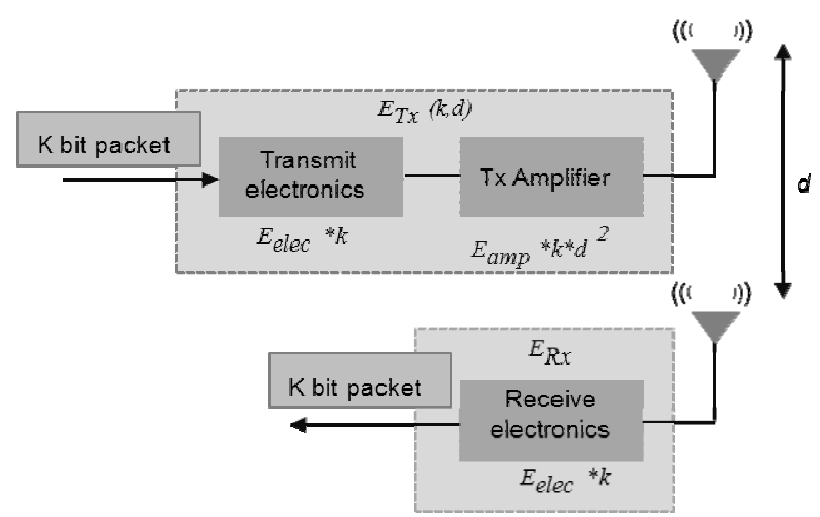

Fig. 4. Radio dissipation model.

The experimental results of ECM are represented in comparison with EER [11] for different criteria. We assumed that the sink is initially located at the center of the designated network field, and moves around with the same pattern and velocity as a sensor node. The sensors are randomly deployed in a $(410 \times 410)$ square meter $(\mathrm{L} \times \mathrm{W})$ area and the transmission range $R$ is $200 \mathrm{~m}$. The sensor nodes move in a random direction with the velocity (2-6) $\mathrm{m} / \mathrm{s}$. This scheme used the user datagram protocol (UDP) as 
the transmission protocol and constant bit rate (CBR) packet with 64 bytes for both data and control data. We assume, a packet drop probability in the ratio of $(0.0-0.2)$.

\section{B. Performance Results}

\section{1) Optimized Number of GNs and CHs in Varying Scale Networks}

In this simulation, the designated network area is varied from $(110 \times 110)$ to $(810 \times 810)$, and 6,400 nodes are gradually deployed in the network. The result in Fig. 5 shows that ECM reduces the significant number of GNs (5.19\%) and CHs (7.53\%), with proper distribution in the entire network. We considered one $\mathrm{CH}$ node in a cluster rather than two $\mathrm{CH}$ nodes, unlike EER. The greater number of $\mathrm{GN}$ and $\mathrm{CH}$ nodes in a network consume more energy for broadcasting more control packets. It is also observed that in EER, the $12.72 \%$ greater number of nodes leads to quicker death than in ECM.

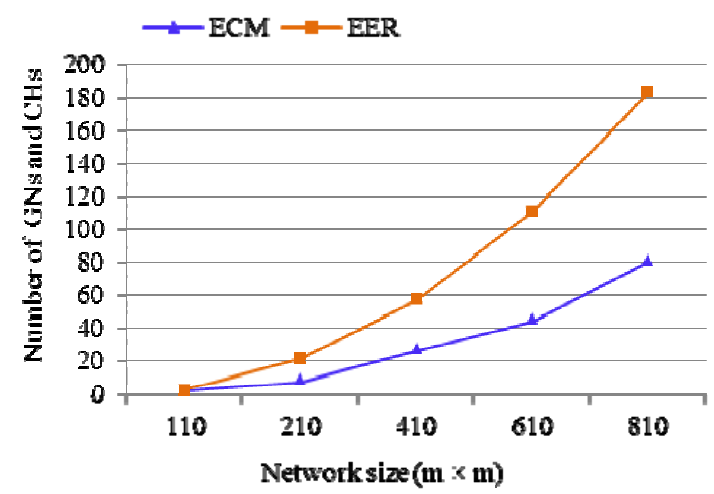

Fig. 5. The numbers of elected $\mathrm{GNs}$ and $\mathrm{CHs}$ in networks of varying scale.

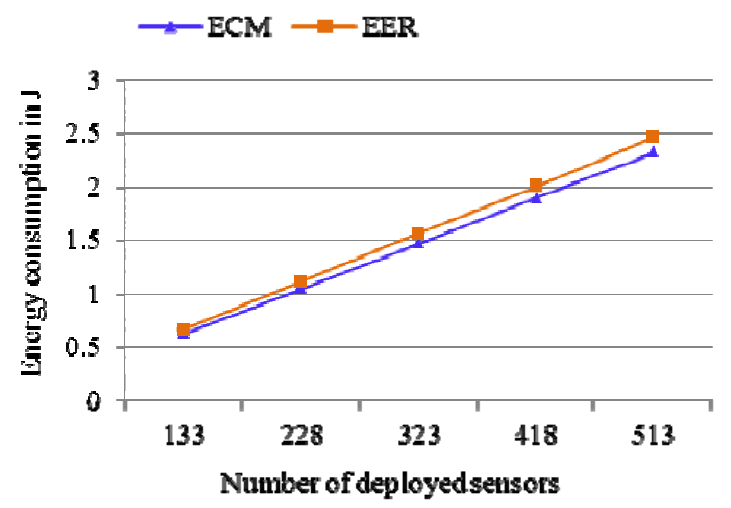

Fig. 6. Energy consumption in the election of gateway node and cluster head.

\section{2) Energy Efficiency in Network Construction}

In the WSN construction and routing field, energy efficiency is one of the goals for reducing the amount of energy required, and to obtain better services in the network lifetime. From the energy efficiency point of view, the network construction cost is shown in Fig. 6. To evaluate the cost, 133 to 513 nodes are randomly deployed in a $(410 \times$ 410) network area in different rounds, as described in Section III. As it is, the energy consumption mainly depends on the number of elected $\mathrm{GN}$ and $\mathrm{CH}$ nodes. ECM consumes $0.025 \%$ less energy in the $\mathrm{GN}$ and $\mathrm{CH}$ election phase due to the smaller number of elected $\mathrm{GN}$ and $\mathrm{CH}$ nodes than that of EER.

\section{3) Idle-listening Energy Dissipation}

Idle-listening is the dominant mode which continuously consumes a sensor's energy. In most existing protocols, such as IEEE 802.11, a power-saving mode (PSM), attempts to reduce the time spent in idle-listening by sleep scheduling. We applied $2 \mathrm{~ms}$ for each time slot in TDMA in its MAC layer and considered 19 frames as a round. It is estimated that, in EER, the normal sensor nodes dissipate $3.62 \%$ more energy than that used ECM due to the two consecutive time slots allocated by two $\mathrm{CHs}$ for a member sensor node in a cluster. The results are shown in Fig. 7.

\section{4) Routing Efficiency}

There are 133 nodes randomly chosen among the deployed sensors to transmit data to the sink in their respective allocated time slots. To calculate the routing cost, we considered 19 clusters (of radius $R=100 \mathrm{~m}$ ) formed in the same area in B.2. In each round, an average of 5 member sensor nodes were connected with their respective $\mathrm{CHs}$ and participated in data forwarding in their allocated time slots, whereas 4 member nodes participated in EER. Fig. 8 shows the overall energy consumption of network construction cost, routing cost, and idle-listening dissipated energy found from our calculations.

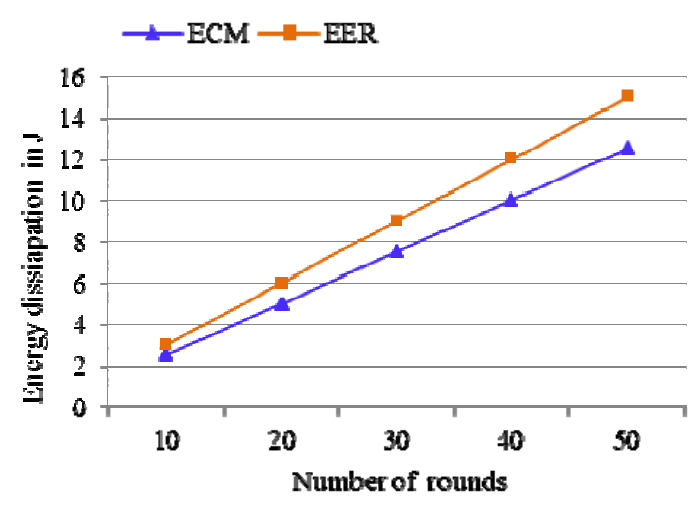

Fig. 7. The energy dissipation in idle-listening. 


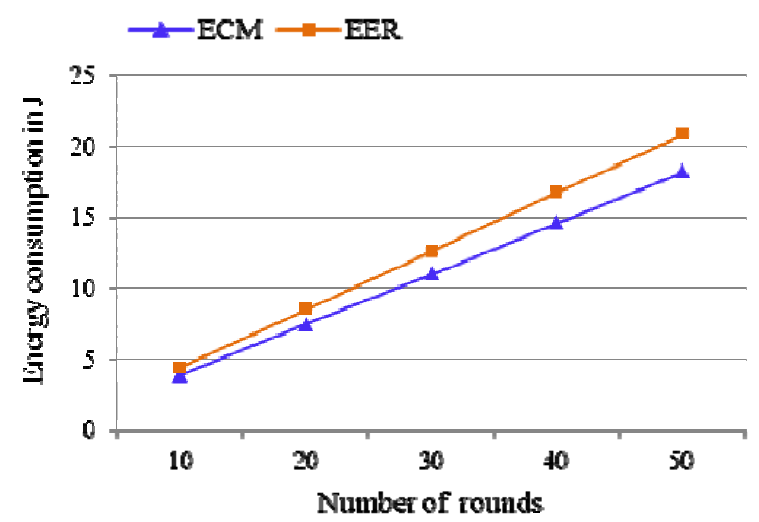

Fig. 8. Overall energy consumption.

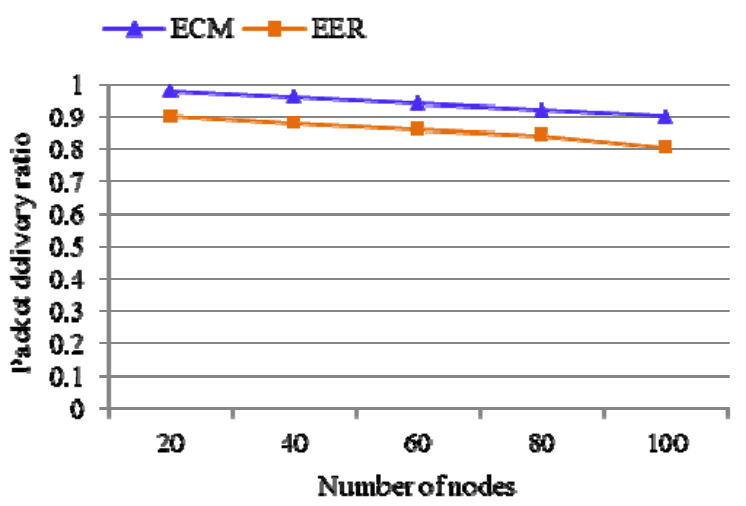

Fig. 9. Packet delivery ratio with respect to network size.

\section{5) Packet Delivery Ratio}

The objectives of WSNs are to perform proper actions based on the received information from the deployed sensors in specific sensing environments. Therefore, the packet reception ratio at the sink plays an important role in WSN applications. To analyze the level, we gradually increased the number of sensors deployed to participate in data forwarding. Fig. 9 shows the received packet ratio at the sink of both ECM and EER. Our proposed scheme outperformed than that of EER due to having minimum number of GNs connected with the $\mathrm{CHs}$ as intermediate nodes for forwarding data to the sink and adaptively recovering the missing data by GN.

\section{CONCLUSIONS}

The static and the mobile nodes in a WSN in a large area will provide a communication bridge between the physical world and the tremendously growing information infrastructure. In this paper, we made use of a hierarchical approach from the initial network construction to the data forwarding to reduce the overall cost, especially from the energy consumption point of view. The proposed gateway node concept for a group of clusters is advantageous for observing the mobile sensors and collecting data from missing nodes. With our work, we observed that, distributing tasks into different nodes in a hierarchical fashion can reduce the energy dissipation for some specific nodes, which in turn can reduce the number of node deaths during the network lifetime. Therefore, we conclude that the proposed scheme is able to lengthen the lifetime of the network effectively. In future work we plan to enhance our scheme for the location-free self-organizing routing as well as data recovery methods in WSNs.

\section{REFERENCES}

[1] H. Luo, Y. Liu, and S. K. Das, "Routing correlated data in wireless sensor networks: a survey,” IEEE Network, vol. 21, no. 6, pp. 40-47, 2007.

[2] Y. Chen and K. Lee, "A routing protocol based on contextawareness for energy conserving in MANETs," International Journal of Maritime Information and Communication Sciences, vol. 5, no. 2, pp. 104-108, 2007.

[3] O. Younis, M. Krunz, and S. Ramasubramanian, "Node clustering in wireless sensor networks: recent developments and deployment challenges," IEEE Network, vol. 20, no. 3, pp. 20-25, 2006.

[4] C. Hur and H. Kim, "Environmental monitoring system for base station with sensor node networks," International Journal of Maritime Information and Communication Sciences, vol. 7, no. 3, pp. 258-262, 2009.

[5] C. E. Perkins and P. Bhagwat, "Highly dynamic destinationsequenced distance-vector routing (DSDV) for mobile computers," Proceedings of the Conference on Communications Architectures, Protocols and Applications, London, UK, pp. 234-244, 1994.

[6] D. B. Johnson and D. A. Maltz, "Dynamic source routing in ad hoc wireless networks," in Mobile Computing, Boston: Kluwer Academic Publishers, pp. 153-181, 1996.

[7] C. E. Perkins and E. M. Royer, "Ad-hoc on-demand distance vector routing," Proceedings of the 2nd IEEE Workshop on Mobile Computing Systems and Applications, New Orleans, LA, pp. 90-100, 1999.

[8] W. R. Heinzelman, A. Chandrakasan, and H. Balakrishnan, "Energy-efficient communication protocol for wireless microsensor networks," Proceedings of the 33rd Annual International Conference on System Sciences, Maui, HI, 2000.

[9] S. Lindsey and C. S. Raghavendra, "PEGASIS: Power-efficient gathering in sensor information systems," Proceedings of IEEE Aerospace Conference, Big Sky, MT, pp. 1225-1130, 2002. 
[10] C. K. Liang and J. D. Lin, "Distributed landmark election and routing protocol for grid-based wireless sensor networks," Proceedings of International Symposium on Parallel and Distributed Processing with Applications, Taipei, Taiwan, pp. 187$191,2010$.

[11] H. K. D. Sarma, A. Kar, and R. Mall, "Energy efficient routing protocol for wireless sensor networks with node and sink mobility," Proceedings of IEEE Sensors Applications Symposium, San Antonio, TX, pp. 239-243, 2011.
[12] Information Sciences Institute. The network simulator (ns-2) [Internet]. Available: http://www.isi.edu/nsnam/ns/.

[13] S. A. B. Awwad, C. K. Ng, N. K. Noordin, and M. F. A. Rasid, "Cluster based routing protocol for mobile nodes in wireless sensor network," Proceedings of International Symposium on Collaborative Technologies and Systems, Baltimore, MD, pp. 233241,2009

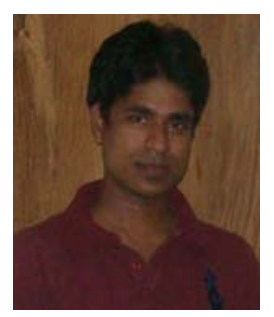

\section{A. S. M. Sanwar Hosen}

received his B.S. degree in computer science and engineering from East West University, Dhaka, Bangladesh, in 2010. Currently, he is a master's student in computer science and engineering at Chonbuk National University, Jeonju, Korea. His current research interests include wireless sensor networks, routing protocols, wireless network security, and green IT.

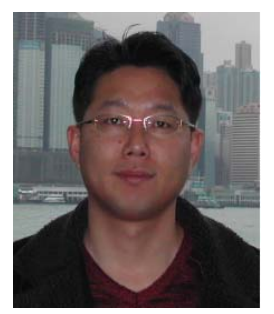

\section{Seung-Hae Kim}

received his B.S. degree in information and communication engineering from Hannam University, Daejeon, Korea, in 1996 and his M.S. degree in information science from Chonbuk National University, Jeonju, Korea, in 2003. He received his Ph.D. degree in information and security engineering from Chonbuk National University, Jeonju, Korea in 2008. Since 1996, he has been with the Korea Institute of Science and Technology Information (KISTI), Daejeon, Korea, where he is currently Head of the Advanced KREONET Service Department. His current research interests include mobile computer communication, security of networks, cloud computing, and big data transfer protocols.

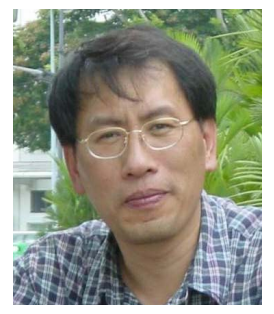

\section{Gi-Hwan Cho}

received his B.S. degree from Chonnam University, Gwangju, Korea, in 1985, and his M.S. degree from Seoul National University, Seoul, Korea, in 1987, both in computer science and statistics. He received his Ph.D. degree in computer science from the University of Newcastle, Newcastle Upon Tyne, England, in 1996. He worked for the Electronics and Telecommunications Research Institute (ETRI), Daejeon, Korea, as a Senior Member of the Technical Staff from Sep. 1987 to Aug. 1997, for the Department of Computer Science at Mokpo National University, Mokpo, Korea, as a Fulltime Lecturer from Sep. 1997 to Feb. 1999. In Mar. 1999, he joined the Division of Computer Science and Engineering at Chonbuk National University, Jeonju, Korea, and he is currently serving as a professor. His current research interests include mobile computing, computer communication, security of wireless networks, sensor networks, and distributed computing systems. 